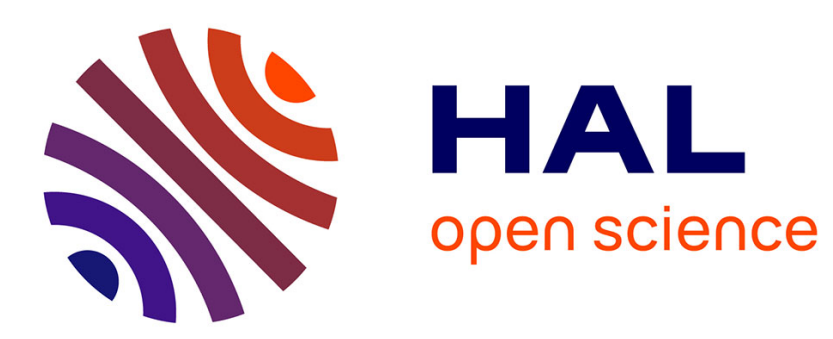

\title{
Quantum chemical topology from tight augmented core densities
}

Julien Pilmé

\section{To cite this version:}

Julien Pilmé. Quantum chemical topology from tight augmented core densities. Journal of Computational Chemistry, 2020, 41 (17), pp.1616-1627. 10.1002/jcc.26204 . hal-02967424

\section{HAL Id: hal-02967424 \\ https://hal.sorbonne-universite.fr/hal-02967424}

Submitted on 14 Oct 2020

HAL is a multi-disciplinary open access archive for the deposit and dissemination of scientific research documents, whether they are published or not. The documents may come from teaching and research institutions in France or abroad, or from public or private research centers.
L'archive ouverte pluridisciplinaire HAL, est destinée au dépôt et à la diffusion de documents scientifiques de niveau recherche, publiés ou non, émanant des établissements d'enseignement et de recherche français ou étrangers, des laboratoires publics ou privés. 


\title{
Quantum Chemical Topology From Tight Augmented Core Densities
}

\author{
Julien Pilmé ${ }^{1}$
}

Correspondence to: Julien Pilmé (E-mail: pilme@lct.jussieu.fr)

\begin{abstract}
Based on parametrized tight Gaussian functions, an efficient and robust methodology designed to restore the effective core potentials electron densities and the inner shells of the electron localization function (ELF) is introduced and tested. Attention is focused on the underlying effects of augmented coreless electron densities on selected quantum topological descriptors computed for a test set of species containing heavy elements such as the emblematic uranyl cation. Also, this paper shows how a proper topology of the electron density can be recovered from semi-empirical Hückel calculations where core densities are missing.
\end{abstract}

Keywords: Quantum Chemical Topology; QTAIM ; Electron localization function; ECP; Core density

I. Introduction. When the nuclear charge grows, the appropriate treatment of molecular systems needs to perform relativistic calculations based on the exact Hamiltonian. However, the relativistic effective core potential (RECP) ${ }^{[1]}$, and especially the pseudopotential $(P P)^{[2]}$, has gained popularity as an approximate quasirelativistic method. The intent is to drastically reduc the computational cost by avoiding the expensive explicit treatment of the chemically inert core electrons of the heavy atoms. Owing to a significant reduction in the number of basis functions and a straightforward form of the Hamiltonian employed, the approach makes it possible to deal with large sized systems while retaining a high degree of reliability for the all-electron calculations. ${ }^{[3,4]}$ However, some troubles in the scalar field of the electron density appear within inner regions where electrons are explicitly replaced by PPs and also in the border regions where the pseudopotential and the all-electron wavefunction coincides. ${ }^{[5-7]}$ For instance, it may lead to either the absence of a bond critical point (CPs) or to the presence of numerous spurious CPs leading to the appearance of nonnuclear maxima and also to unreasonable electronic properties even outside of the core regions. Similar pathologies in the electron localization function $(E L F)^{[8]}$ field can be also observed. Hence, it makes quantum chemical topology analysis very difficult without a recovery of core regions. Amongst other things, it was shown that the interatomic flux null surfaces of the quantum theory of atoms in molecules (QTAIM) ${ }^{[9,10]}$ can become very wrong. Thus, substantial numerical errors are made when integrating the core regions of heavy atoms and erroneous energetic contributions are obtained in the context of the interacting quantum atoms (IQA) $)^{[11,12]}$ method. As is generally known, these failures can be cured by augmenting the valence density with electron density functions of atomic core electrons. ${ }^{[5,13-}$ 15]

In the scientific literature, there are several ways for correcting the coreless densities $^{[5,11,13-16]}$, but typically, the additional atomic density is generated from new core orbitals obtained from additional $a b$ initio calculations, the latter being orthogonalized to the valence ones. Several implementations have been proposed and data libraries are also available. ${ }^{[17]}$ However, this process produces new orbitals generated from a different level of theory and, the method presents also some compatibility issues with the relativistic wavefunction where spinors are used instead of molecular orbitals. Spinors are typically two- or four-component complex functions produced by relativistic calculations. 
It has been reported in the literature ${ }^{[16]}$ that it may be possible to use a crude core density by considering only a tight normalized frozen s-type Gaussian function,

$$
\rho\left(\mathbf{r} ; \mathbf{R}_{\mathrm{A}}\right)_{\mathrm{A}}^{\text {core }}=8 Z_{\mathrm{A}}^{\text {core }} e^{-4 \pi\left(\mathbf{r}-\mathbf{R}_{\mathrm{A}}\right)^{2}}
$$

which integrates to the number of ECP-modeled core electrons $Z_{A}^{\text {core }}$ for the atom $A$. In the case of heavy elements where small-core ECPs are used, the performance of this function to restore the core density remains impressive compared to more realistic methods previously discussed. However, for large-core ECPs or lighter elements, the performance appears clearly less accurate. ${ }^{[16]}$

In this work, we explore how parametrized single s-type functions could be used to efficiently fulfill the coreless electron density for all atoms of the periodic table, whether small-core or large-core ECPs, at any level of theory, including quasirelativistic ECP. The methodology needs also to be tailored to a crude analysis of three-dimensional grids where the electron density has been only calculated for each grid point. In addition, attention has also been focused on restoring the inner shells of the electron localization function (ELF).

\section{Sketch of Quantum Chemical Topology.} In this article, we assume that the reader is familiar with the quantum chemical topology (QCT) of scalar fields because numerous presentations of the method and many applications have already been published in the literature. ${ }^{[18-24]}$ Briefly, the purpose is to answer general questions about the chemical bonding in molecules or solids, and predict or explain the chemical reactivity trends. In a QCT analysis, a partitioning of the molecular space into subsystems (basins) is achieved by applying the theory of dynamical gradient systems to the properties of the scalar function. The basins are localized around the maxima of the function and are separated by the zero flux surfaces. In the quantum theory of atoms in molecules the function considered is the one electron density and these basins are associated with each of the atoms in the molecule. The topology of the gradient field is characterized by its critical points ( $C P)$, where the gradient of the electron density is zero, and by their connectivity. CP can be either maxima $(3,-3)$, minima $(3,3)$ or saddle points. Among the saddle points, the termed bond critical point $(3,-1)(\mathrm{BCP})$ has a crucial role because it connects two maxima by only a trajectory of the gradient field (the bond path). Further insights into the chemical bond can be gained by means of topological descriptors calculated at the BCP. More elaborate scalar fields can be used such as the electronic localization function of Becke and Edgecombe ${ }^{[8,25]}$ which is typically interpreted as a signature of the electronic-pair distribution. ${ }^{[26]}$

III. Theory. Overall, the coreless electronic density $\rho(\mathbf{r})^{\text {val }}$ arising from a pseudopotential calculation can be augmented by adding allelectron core atomic density $\rho(\mathbf{r})^{\text {core }}$ as follows:

$$
\rho(\mathbf{r})=\rho(\mathbf{r})^{\text {core }}+\rho(\mathbf{r})^{\mathrm{val}}
$$

where,

$$
\rho(\mathbf{r})^{\mathrm{core}}=\sum_{\mathrm{A}} \rho\left(\mathbf{r} ; \mathbf{R}_{\mathrm{A}}\right)_{\mathrm{A}}^{\text {core }}
$$

and

$$
\rho(\mathbf{r})^{\mathrm{val}}=\sum_{\mathrm{i}}^{\mathrm{occ}} \mathrm{n}_{\mathrm{i}}\left|\varphi_{\mathrm{i}}(\mathbf{r})\right|^{2}
$$

and $n_{i}$ is the occupation of the molecular orbital $\varphi_{i}(\mathbf{r})$. In a quasirelativistic context, molecular orbitals are replaced by two-component complex single-particle functions known as spinors, $\varphi_{i}(r)$ the expansion coefficients $c_{i}$ being complex and typically determined within the SCF procedure. ${ }^{[27,28]}$ The electron density needs to be computed as follows:

$$
\rho(\mathbf{r})^{\mathrm{val}}=\sum_{\mathrm{i}}^{\text {occ }} \varphi_{\mathrm{i}}^{\dagger}(\mathbf{r}) \varphi_{\mathrm{i}}(\mathbf{r})
$$

Note that the knowledge of molecular orbitals or molecular spinors is not mandatory in order 
to perform a QCT analysis because $\rho(\boldsymbol{r})^{\mathrm{val}}$ can only be known through a three dimensional grid directly produced by the quantum chemistry program.

Preserving the constraint to integrate core electrons $Z_{A}^{\text {core }}$ in the whole molecular space, the core density can be defined through a 1s- type tight Gaussian function as follows:

$$
\rho\left(\mathbf{r} ; \mathbf{R}_{\mathrm{A}}\right)_{\mathrm{A}}^{\text {core }}=\rho_{\mathrm{A}}^{0} e^{-\pi \alpha^{2}\left(\mathbf{r}-\mathbf{R}_{\mathrm{A}}\right)^{2}}
$$

where $\rho_{A}^{0}$ is the value of the core density at the nuclear location $\mathbf{R}_{\mathrm{A}}$ of an atom $A$ and $\alpha$ is a positive exponent somehow related to the width of the core electron function in the vicinity of the nucleus. Then, $\alpha=\left(\frac{\rho_{\mathrm{A}}^{0}}{Z_{\mathrm{A}}^{\text {core }}}\right)^{\frac{1}{3}}=\frac{1}{2 \sigma^{2}}$ and $\sigma=\frac{1}{\alpha \sqrt{2 \pi}}$ the standard deviation. For the simplistic case previously discussed in the literature ${ }^{[16]}, \rho_{A}^{0}$ was frozen to $8 Z_{A}^{\text {core }}$ which gives $\alpha=2$ and $\sigma=\frac{1}{2 \sqrt{2 \pi}}$. The core density function goes to zero in the valence regions which means that the total augmented density $\rho(\mathbf{r})$ tends to coincide with the coreless valence density $\rho(r)^{\text {val }}$. Hence, the spatial extension of the core electron density depends on $\rho_{\mathrm{A}}^{0}$. When ECPs are used (small- or large-core), finding a suitable value of $\rho_{\mathrm{A}}^{0}$ for each atom of a molecule remains a challenge. In addition, the integrated core density over the full atomic QTAIM volume needs also to be constrained to $Z_{A}^{\text {core }}$ for each atom A.

As discussed above, $\rho(\mathbf{r})^{\text {val }}$ exhibits numerous spurious critical points because its gradient can accidentally become almost zero in the border core/valence regions. The number of these points shows a tendency to increase with the size of the ECP core. Thus, the gradient of the augmented density field needs to be corrected for its discrepancies. Interestingly, as illustrated on Figure 1 for the $\mathrm{GeH}_{4}$ molecule $\left(T_{d}\right)$ using a ECP large-core, the spurious critical points of $\rho(\mathbf{r})^{\text {val }}$ appear roughly confined within a so-called $\beta$-sphere of radius $\mathrm{R}_{\beta}=0.29 \mathrm{bohr}$ centered on the Ge nucleus. ${ }^{[11,29]}$

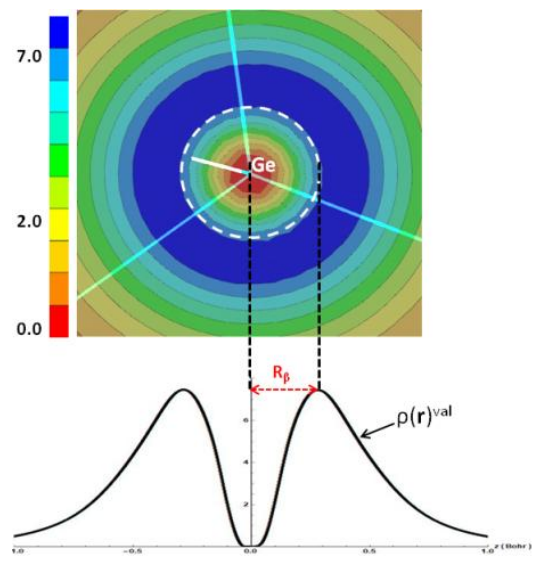

Figure 1: Electron density plot $\rho(\mathbf{r})^{\text {val }}\left(\right.$ e.bohr $\left.r^{-3}\right)$ depicted in the $x z$ plane of the $\mathrm{GeH}_{4}$ molecule $\left(T_{d}\right)$ optimized at the Hartree-Fock level of theory using a CRENBL ECP (largecore) and its respective basis set for $\mathrm{Ge}$ atom and the 6$311 \mathrm{G}(\mathrm{d}, \mathrm{p})$ basis set for $\mathrm{H}$. Core parameters: $\mathrm{Z}_{\mathrm{Ge}}^{\text {core }}=18 \mathrm{e}$, $\mathrm{R}_{\beta}=0.290$ bohr. $\rho_{\beta}=7.36$ e.bohr $^{-3}$.

As a reminder, a $\beta$-sphere is a spherical volume that is fully enclosed within the topological QTAIM basin volume at whose nucleus it is centered. ${ }^{[30,31]}$ Most of the time, the $\beta$-sphere is defined as the largest sphere that is fully contained within the atomic basin. Thus, its radius is enough large, around $90 \%$ of the distance between the nucleus and the nearest bond critical point. In the beginning ${ }^{[29]}$, the $\beta$ sphere was mainly used to easily assign the gradient paths evolving within the space of the topological atom. Later, since the electron density exhibits very high values in the vicinity of the nucleus, it was shown ${ }^{[31]}$ that it remains numerically advantageous to keep the sphere as the most suitable object for fully numerical or analytical integrations.

As noted earlier ${ }^{[11]}$, whatever the PP used for a given atom, a $\beta$-sphere enclosing the spurious points can be always found. When the distance from the nucleus is more than $R_{\beta}$, the topology of the valence density is rather consistent with the corresponding all-electron calculation. As shown on Figure 1, the electron density on the $\beta$-sphere remains almost unchanged around an average value termed here as $\rho_{\beta}$. Hence, a suitable value for $\rho_{\mathrm{A}}^{0}$ can be found if the width of the atomic Gaussian function is lined up with 
$R_{\beta}$. In other words, the following equation has to be verified for a given $R_{\beta}$ :

$$
\mathrm{W}\left(\rho_{\mathrm{A}}^{0}\right)=\rho_{\mathrm{A}}^{0} \mathrm{e}^{-\pi\left(\frac{\rho_{\mathrm{A}}^{0}}{Z_{\mathrm{A}}^{\text {core }}}\right)^{\frac{2}{3}} \mathrm{R}_{\beta}{ }^{2}}=\rho_{\beta}
$$

Because the function involved in the equation (2) has a typical transcendental form ${ }^{[32]}$, it needs to be numerically solved. As shown in Figure 2, the function $\mathrm{W}\left(\rho_{\mathrm{A}}^{0}\right)$ reaches a maxima for a given $\rho_{A}^{0}=\rho_{A}^{0, \text { Max }}$ such as:

$$
\rho_{\mathrm{A}}^{0, \mathrm{Max}}=\frac{3}{2} \sqrt{\frac{3}{2 \pi^{3}}} \frac{\mathrm{Z}_{\mathrm{A}}^{\text {core }}}{\mathrm{R}_{\beta}{ }^{3}}
$$

When $\rho_{\mathrm{A}}^{0}$ goes to large values, the core density goes to zero.

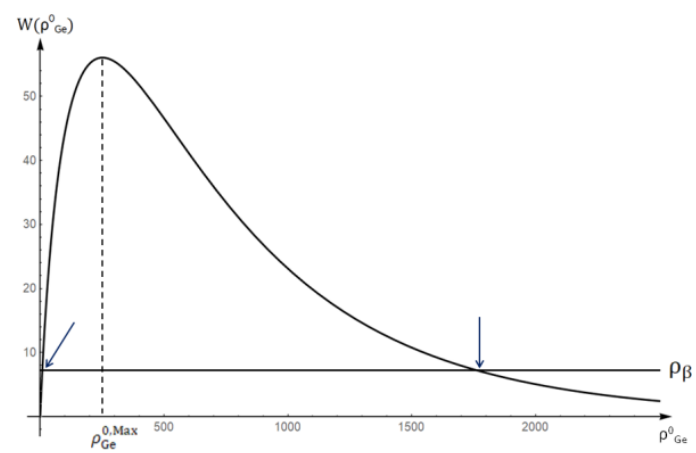

Figure 2. Plot of $\mathrm{W}\left(\rho_{\mathrm{Ge}}^{0}\right)$ according to $\rho_{\mathrm{Ge}}^{0}$ for $\mathrm{GeH}_{4}$ molecule optimized at the Hartree-Fock level of theory. The CRENBL ECP (large-core) and its respective basis set have been employed for Ge while the $6-311 G(d, p)$ basis set has been used for $\mathrm{H}$.

Core parameters for $\mathrm{Ge}: \mathrm{Z}_{\mathrm{Ge}}^{\text {core }}=18 \mathrm{e}, \mathrm{R}_{\beta}=0.290 \mathrm{bohr}$, $\rho_{\beta}=7.36$ e.bohr ${ }^{-3}$ and $\rho_{\mathrm{Ge}}^{0, \mathrm{Max}}=243.49$ e.bohr $^{-3}$.

There is always a suitable value where $\rho_{A}^{0}=\rho_{\beta}$ can be easily found beyond the maxima when the function monotonically decreases. Scheme 1 displays the algorithm that gives a suitable value of $\rho_{\mathrm{A}}^{0}$ larger than $\rho_{\mathrm{A}}^{0, \text { Max }}$ :

$$
\begin{aligned}
& \rho_{A}^{0}=\rho_{A}^{0, \text { Max }} \text { and } W\left(\rho_{A}^{0}\right)=\rho_{A}^{0} \mathrm{e}^{-\pi \alpha^{2} R_{\beta}{ }^{2}} \\
& \text { While }\left(W\left(\rho_{A}^{0}\right)>\rho_{\beta}\right): \\
& \qquad \begin{array}{l}
\rho_{A}^{0}=\rho_{A}^{0}+\text { step } \\
a=\left(\frac{\rho_{A}^{0}}{Z_{A}^{\text {core }}}\right)^{\frac{1}{3}} \text { and } W\left(\rho_{A}^{0}\right)=\rho_{A}^{0} \mathrm{e}^{-\pi \alpha^{2} R_{\beta}^{2}}
\end{array}
\end{aligned}
$$

Scheme 1: Algorithm given the suitable value $\rho_{\mathrm{A}}^{0}$

The step needs to be as small as possible. Practically, a value of 0.001 e.bohr ${ }^{-3}$ has been used in this work. Once, $\rho_{A}^{0}$ is found, the core density function is fully defined. For instance as illustrated in Figure 3 for the $\mathrm{Au}\left({ }^{2} \mathrm{~S}\right)$ atom $(\mathrm{Z}=79$, $Z_{A u}^{\text {core }}=60$ electrons), the augmented density $\rho(r)$ does not show any spurious points and fits very well with both $\rho(r)^{\text {val }}$ in the valence regions and with $\rho(r){ }^{\text {core }}$ in the inner regions. Some tests for various free atoms and molecules are presented in the next section. Effects of small- and largecore ECPs have been evaluated.

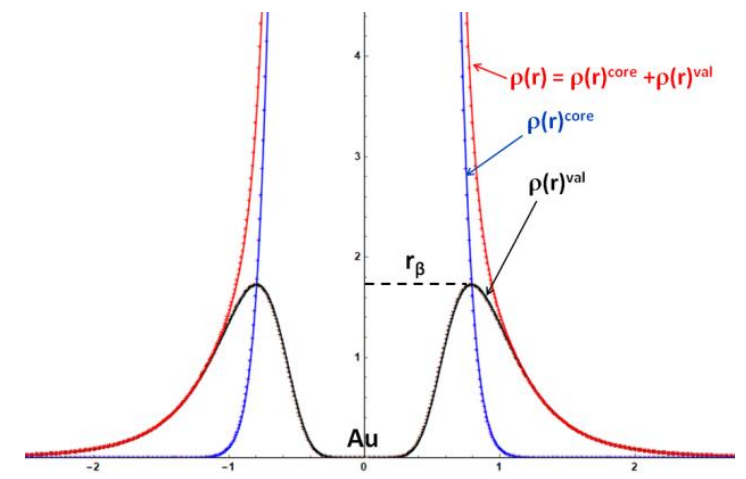

Figure 3: Core, valence and augmented electron densities plots along the $z$ axis (bohr) obtained for the $A u\left({ }^{2} S\right)$ atom calculated at the B3LYP/ aug-cc-pVTZ-PP level of theory. Color code: black: $\rho(\boldsymbol{r})^{\text {val }}$, blue: $\rho(\mathbf{r})^{\text {core }}$ and red: augmented density $\rho(\mathbf{r})=\rho(\mathbf{r})^{\text {core }}+\rho(\mathbf{r})^{\text {val }}$. Core parameters: $Z_{A u}^{\text {core }}=60$ e, $R_{\beta}=0.806$ bohr and $\rho_{\beta}=1.723$ e.bohr ${ }^{-3}$.

Finally, the core population $\mathrm{N}_{\mathrm{A}}^{\beta}$ can be computed over the volume of any $\beta$-sphere having a radius $R_{\beta}$. This $\beta$-sphere is enclosed in the basin volume $\Omega_{\mathrm{A}}$ centered on an atom A.

$$
\begin{aligned}
N_{A}^{\beta}\left(R_{\beta}\right) & =\int_{R_{A}}^{R_{A}+R_{\beta}} \rho\left(r ; R_{A}\right)_{A}^{\text {core }} d r \\
& =Z_{A}^{\text {core }}\left[\operatorname{Erf}\left(\alpha \sqrt{\pi} R_{\beta}\right)-2 R_{\beta} \alpha \mathrm{e}^{-\pi \alpha^{2} R_{\beta}^{2}}\right]
\end{aligned}
$$

Note that because the exponential function quickly goes to zero when $R^{\beta}{ }_{A}$ increases, $N_{A}^{\beta}$ quickly reachs $\mathrm{Z}_{\mathrm{A}}^{\text {core }}$.

The Gradient of the electron density. In addition to the lack of maxima at nuclear positions when electrons are replaced by PPs, the gradient vectors of the electron density 
$\nabla \rho(\mathbf{r})^{\text {val }}$ becomes accidentaly close to zero in the vicinity of nuclear cores entailing the presence of some spurious saddle points. This feature is a serious problem because gradient vectors are represented through the trajectories (also referred as gradient paths) characterizing the topological properties of the gradient field. The addition of core density cures these pathologies and the augmented gradient vector becomes defined as,

$$
\begin{aligned}
\nabla \rho(\mathbf{r}) & =\nabla \rho(\mathbf{r})^{\text {core }}+\nabla \rho(\mathbf{r})^{\mathrm{val}} \\
& =\sum_{\mathrm{A}} \nabla \rho\left(\mathbf{r} ; \mathbf{R}_{\mathrm{A}}\right)_{\mathrm{A}}^{\text {core }}+\nabla \rho(\mathbf{r})^{\mathrm{val}}
\end{aligned}
$$

where

$$
\nabla \rho\left(\mathbf{r} ; \mathbf{R}_{\mathrm{A}}\right)_{\mathrm{A}}^{\text {core }}=-2 \pi \alpha^{2} \rho\left(\mathbf{r} ; \mathbf{R}_{\mathrm{A}}\right)_{\mathrm{A}}^{\text {core }}\left(\mathbf{r}-\mathbf{R}_{\mathrm{A}}\right)
$$

As expected, when $\rho\left(\mathbf{r} ; \mathbf{R}_{\mathrm{A}}\right)_{\mathrm{A}}^{\text {core }}=\rho_{\beta}$ (regions where spurious points are located), $\left|\nabla \rho^{\mathrm{val}}(\mathbf{r})\right|$ becomes close to zero and therefore,

$$
\nabla \rho(\mathbf{r}) \approx \nabla \rho(\mathbf{r})^{\text {core }}=-2 \pi \alpha^{2} \rho_{\beta} \mathrm{R}_{\beta}
$$

The Laplacian of the electron density. The Laplacian plays also a key role in quantum chemical topology because it is directly related to the virial theorem. ${ }^{[3,34]}$ Indeed, the Laplacian vanishes when it is integrated over the whole space or over the whole atomic basin. This property ensures that the total kinetic energy and the atomic kinetic energy are unambiguous defined. Moreover, the Laplacian of the density is a powerful descriptor of the chemical bond because a large negative value at the BCP indicates a local concentration of charge which reveals a typical shared-shell interaction. ${ }^{[35]}$ The augmented Laplacian is expressed as:

$$
\begin{aligned}
\nabla^{2} \rho(\mathbf{r}) & =\nabla^{2} \rho(\mathbf{r})^{\text {core }}+\nabla^{2} \rho(\mathbf{r})^{\text {val }} \\
& =\sum_{\mathrm{A}} \nabla^{2} \rho\left(\mathbf{r} ; \mathbf{R}_{\mathrm{A}}\right)_{\mathrm{A}}^{\text {core }}+\nabla^{2} \rho(\mathbf{r})^{\mathrm{val}}
\end{aligned}
$$

where

$\nabla^{2} \rho\left(\mathbf{r} ; \mathbf{R}_{\mathrm{A}}\right)_{\mathrm{A}}^{\text {core }}=2 \pi \alpha^{2} \rho\left(\mathbf{r} ; \mathbf{R}_{\mathrm{A}}\right)_{A}^{\text {core }}\left[2 \pi \alpha^{2}\left(\mathbf{r}-\mathbf{R}_{\mathrm{A}}\right)^{2}-3\right]$

The integrated core laplacian over the volume of any $\beta$-sphere of radius $R_{\beta}$ enclosed in a basin volume $\Omega_{\mathrm{A}}$ centred on an atom $\mathrm{A}$ is then defined as follows:

$$
\begin{aligned}
\mathrm{L}_{\mathrm{A}}^{\beta}\left(\mathrm{R}_{\beta}\right) & =\int_{\mathrm{R}_{\mathrm{A}}}^{\mathrm{R}_{\mathrm{A}}+\mathrm{R}_{\beta}} \nabla^{2} \rho\left(\mathbf{r} ; \mathbf{R}_{\mathrm{A}}\right)_{\mathrm{A}}^{\text {core }} \mathrm{d} \mathbf{r} \\
& =-8 \pi^{2} \alpha^{5} \mathrm{Z}_{\mathrm{A}}^{\text {core }}\left(\mathrm{R}_{\beta}\right)^{3} \mathrm{e}^{-\pi \alpha^{2} \mathrm{R}_{\beta}{ }^{2}}
\end{aligned}
$$

As expected, this quantity goes quickly to zero when $\mathrm{R}_{\beta}$ increases.

The Kinetic positive energy density (KED). The augmented local positive definite KED $G(\mathbf{r})$ can be also defined from valence and core contributions. $G(\mathbf{r})$ can be trivially separated into two parts as follows:

$$
\mathrm{G}(\mathbf{r})=\tau(\mathbf{r})^{\text {core }}+\tau(\mathbf{r})^{\mathrm{val}}
$$

where

$$
\tau(\mathbf{r})^{\mathrm{val}}=\sum_{\mathrm{i}}^{\text {occ. }} \nabla \varphi_{\mathrm{i}}^{\dagger}(\mathbf{r}) \nabla \varphi_{\mathrm{i}}(\mathbf{r})
$$

In this work, $\tau(r))^{\text {val }}$ corresponds to the KED computed at the Hartree-Fock level or whether for a Kohn-Sham fictitious non interacting system, $\varphi_{\mathrm{i}}$ being molecular orbitals or complex spinors defined in a quasirelativistic context. However, if orbitals or spinors are unknown, the KED can be extracted from the ELF functional form regardless of the quantum chemistry program (DFT calculation) used to generate the grid points. Thus, the valence kinetic energy density $\tau(\mathbf{r})^{\text {val }}$ is defined from $\mathrm{ELF}^{[25]}$ in its functional form for each point of the grid as follows:

$$
\tau^{\mathrm{val}}(\mathbf{r})=\left(\frac{1}{\operatorname{ELF}(\mathbf{r})^{\mathrm{val}}}-1\right)^{1 / 2} c_{\mathrm{f}}\left(\rho(\mathbf{r})^{\mathrm{val}}\right)^{5 / 3}+\frac{1}{8} \frac{\left|\nabla \rho(\mathrm{r})^{\mathrm{val}}\right|^{2}}{\rho(\mathbf{r})^{\mathrm{val}}}
$$

with $\mathrm{c}_{\mathrm{f}}=\frac{3}{10}\left(3 \pi^{2}\right)^{2 / 3}$.

For the special case where $Z_{A}^{\text {core }}=2$,

$$
\tau^{\text {core }}(\mathbf{r})=\sum_{A} \tau\left(\mathbf{r} ; \mathbf{R}_{\mathrm{A}}\right)^{w c}=\sum_{A} \frac{\left|\nabla \rho\left(\mathbf{r} ; \mathbf{R}_{\mathrm{A}}\right)_{A}^{\text {core }}\right|^{2}}{8 \rho\left(\mathbf{r} ; \mathbf{R}_{\mathrm{A}}\right)_{\mathrm{A}}^{\text {core }}}
$$

${ }^{[36]}$ is the von Weizsäcker kinetic energy core density, i.e. the kinetic energy of a density 
model system in which the antisymmetry is switched off.

When $Z_{\mathrm{A}}^{\text {core }}>2$, the functional form proposed by Kirzhnits and its coworkers ${ }^{[37,38]}$ and latter revivified by Ayers and coworkers ${ }^{[39]}$ can be used to restore the KED in the inner regions:

$\tau^{\text {core }}(\mathbf{r})=\sum_{\mathrm{A}} \tau\left(\mathbf{r} ; \mathbf{R}_{\mathrm{A}}\right)_{\mathrm{A}}^{\text {core }}$

with

$$
\begin{array}{r}
\tau\left(\mathbf{r} ; \mathbf{R}_{\mathrm{A}}\right)_{\mathrm{A}}^{\text {core }}=\mathrm{W}_{\mathrm{A}}\left(\mathbf{r} ; \mathbf{R}_{\mathrm{A}}\right) \tau\left(\mathbf{r} ; \mathbf{R}_{\mathrm{A}}\right)^{\mathrm{WC}}+\left(1-\mathrm{W}_{\mathrm{A}}\left(\mathbf{r} ; \mathbf{R}_{\mathrm{A}}\right)\right) \\
\left(\tau\left(\mathbf{r} ; \mathbf{R}_{\mathrm{A}}\right)^{\mathrm{TFC}}+\frac{\tau\left(\mathbf{r} ; \mathbf{R}_{\mathrm{A}}\right)^{\mathrm{WC}}}{9}+\frac{\nabla^{2} \rho\left(\mathbf{r} ; \mathbf{R}_{\mathrm{A}}\right)_{\mathrm{A}}^{\text {core }}}{6}\right)
\end{array}
$$

where $\tau\left(\mathbf{r} ; \mathbf{R}_{\mathrm{A}}\right)^{\mathrm{TFC}}=\mathrm{c}_{\mathrm{f}}\left(\rho\left(\mathbf{r} ; \mathbf{R}_{\mathrm{A}}\right)_{\mathrm{A}}^{\text {core }}\right)^{5 / 3}$ is the corresponding kinetic energy core density for a homogeneous electron gas and

$$
\mathrm{W}_{\mathrm{A}}\left(\mathbf{r} ; \mathbf{R}_{\mathrm{A}}\right)=\mathrm{e}^{-\ln (2)\left(\frac{\left|\mathbf{r}-\mathbf{R}_{\mathrm{A}}\right| \mathrm{Z}_{\mathrm{A}}}{\ln (2)}\right)^{2}}
$$

is a weight function close to one in the vicinity of nuclei and goes to zero elsewhere.

Electron Localization Function. During the last twenty five years, the ELF topological analysis ${ }^{[26]}$ has been used for studying of the bonding schemes in molecules and solids, or for rationalizing chemical reactivity. ${ }^{[40]}$ In its original formulation ${ }^{[8]}$ ELF relies on the Laplacian of the conditional same spin pair probability scaled by the homogeneous electron gas kinetic energy density, ELF being typically interpreted as a signature of the electron-pair distribution. From 1992, this formulation has been extended in a functional form, and rationalized in terms of the local excess kinetic energy due to Pauli repulsion. ${ }^{[25]}$ However, by analogy to the spindensity functional theory, a decomposition in terms of spin densities (spin-polarized ELF) was also proposed. ${ }^{[41]}$ In a similar manner, various orbital decompositions have also been proposed such as $E F_{\sigma / \pi}$ or $E_{\text {core/valence }}$ respectively conducted from $\rho_{\sigma} / \rho_{\pi}$ or $\rho_{\text {core }} / \rho_{\text {valence }}$ groups of molecular orbitals. ${ }^{[42,43]}$ In this latter form, the augmented ELF kernel is defined from both core and valence KED as well as from both core and valence electron densities as follows:

$$
\chi(\mathbf{r})=\frac{\tau(\mathbf{r})^{\text {core }}-\frac{1}{8} \frac{\left|\nabla \rho(\mathbf{r})^{\text {core }}\right|^{2}}{\rho(\mathbf{r})^{\text {core }}}+\tau^{\text {val }}(\mathbf{r})-\frac{1}{8} \frac{\left|\nabla \rho(\mathbf{r})^{\text {val }}\right|^{2}}{\rho(\mathbf{r})^{\text {val }}}}{c_{\mathrm{f}}\left(\rho(\mathbf{r})^{\text {core }}+\rho(\mathbf{r})^{\mathrm{val}}\right)^{5 / 3}}
$$

and $\operatorname{ELF}=\frac{1}{1+\chi^{2}(\mathbf{r})}$

The augmented ELF kernel can be also extracted from both $\operatorname{ELF}^{\mathrm{val}}(\mathbf{r})$ and electron density $\rho(\mathbf{r})^{\mathrm{val}}$ grid points as:

$$
\chi(\mathbf{r})=\frac{\tau(\mathbf{r})^{\text {core }}-\frac{1}{8} \frac{\left|\nabla \rho(\mathbf{r})^{\text {core }}\right|^{2}}{\rho(\mathbf{r})^{\text {core }}}+\left[\frac{1}{\operatorname{ELF}(\mathbf{r})^{\text {val }}-1}\right]^{1 / 2} c_{f}\left(\rho(\mathbf{r})^{\text {val }}\right)^{5 / 3}}{c_{f}\left(\rho(\mathbf{r})^{\text {core }}+\rho(\mathbf{r})^{\text {val }}\right)^{5 / 3}}
$$

Hence, as illustrated in Figure 4, it can be noticed that the augmented ELF plots calculated along the $z$ axis for $C\left({ }^{3} \mathrm{P}\right)$ and $\mathrm{Br}\left({ }^{2} \mathrm{~S}\right)$ atoms using the aug-cc-pVTZ-PP basis set (ECP small-core, $Z_{A}^{\text {core }}=10$ e) and the aug-cc-pVTZ basis set display very similar profiles, appearing almost isotopological in the valence regions since the location and properties of critical points (local minima and maxima) are almost the same.

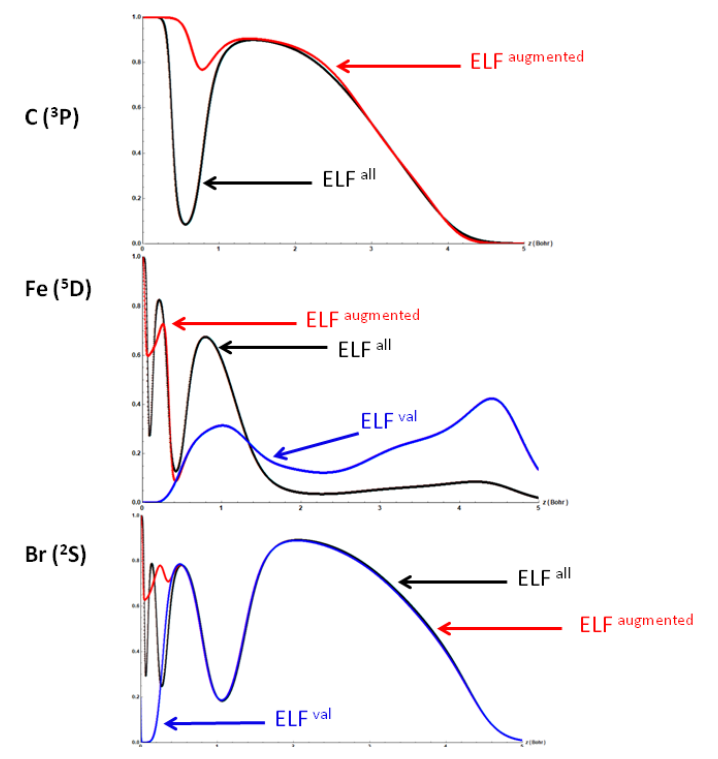

Figure 4: Augmented ELF plots of $C\left({ }^{3} \mathrm{P}\right), \mathrm{Br}\left({ }^{2} \mathrm{~S}\right)$ and $\mathrm{Fe}\left({ }^{5} \mathrm{D}\right)$ atoms along the $z$ axis computed at the B3LYP level of 
theory. The aug-cc-pVQZ-PP (small-core) basis sets have been employed for $\mathrm{Br}$ while the CRENBS ECP (large-core) and its respective basis set have been employed for the $C$ and the Fe atoms. All-electron basis set aug-cc-pVQZ is also displayed for comparison only. $C\left({ }^{3} \mathrm{P}\right)$ parameters: $Z_{C}^{\text {core }}=2$ electrons, $R_{\beta}=0.63$ bohr, $\rho_{C}^{0}=9.29$ e.bohr ${ }^{-3}$; $\mathrm{Br}\left({ }^{2} \mathrm{~S}\right)$ small-core parameters: $Z_{\mathrm{Br}}^{\text {core }}=10$ electrons, $R_{\beta}=$ 0.29 bohr, $\rho_{\mathrm{Br}}^{0}=399.71$ e.bohr ${ }^{-3} ; \mathrm{Fe}\left({ }^{5} \mathrm{D}\right)$ large-core parameters: : $Z_{\mathrm{Fe}}^{\text {core }}=18$ electrons, $\mathrm{R}_{\beta}=0.38$ bohr, $\rho_{\mathrm{Fe}}^{0}=$ 860.61 e. $^{\text {bohr }}{ }^{-3}$.

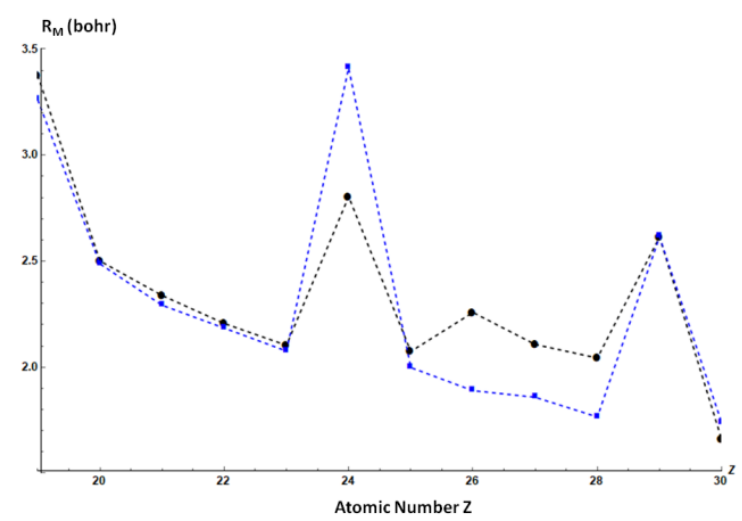

Figure 5. The ELF radius (Bohr) of the $M$ shell calculated at the B3LYP level of theory. Color code: Blue: $6-311+G(3 \mathrm{df})$ basis set. Black: LanL2TZ(f) and its respective basis set.

In any case, the ELF topology is partially restored within the core regions, notably for the maxima located on the nuclear location ( $E L F=1)$. In addition, all the minima associated with radii of inner shells ( $K$ and $L$ minima) ${ }^{[41]}$ are well restored, whether the small-core $\operatorname{ECP}(\mathrm{C}, \mathrm{Br})$ or the large-core ECP (Fe). In the valence regions, the augmented ELF plot obtained for both small- and large-core ECP coincides with the $\mathrm{ELF}^{\mathrm{val}}(\mathbf{r})$ plot for which the core is not rebuilt. As observed in Figure 4, even for two different basis sets (aug-cc-pVQZ and CRENBS), the location of ELF minima of the Fe atom $\left({ }^{5} D\right)$ are almost unchanged whether they are inner atomic regions or the valence regions. For example, the minima associated with L-shell calculated at the B3LYP/CRENBS level of theory was found at 0.426 bohr from the nuclear location while the same minima calculated at the B3LYP/aug-cc-pVQZ level of theory was found at 0.427 bohr from the nuclear location. The calculated radius of the $M$ shell, when $Z$ is restricted between 19 and 36, appears rather reasonable by comparison with similar calculations obtained using the $6-311+G(3 d f)$ basis (see Figure 5). Note that a typical peak of the $\mathrm{M}$ shell radius observed for both $\mathrm{Cr}$ and $\mathrm{Cu}$ atoms is in agreement with previous calculations. $^{[41]}$

Some calculations of ELF radii have also been reported in Table S1 and displayed in Figures S1 and S2. We note that $\mathrm{K}, \mathrm{L}$, and $\mathrm{M}$ shells are adequately reproduced until $\mathrm{Z}=36$. For heavier atoms $(Z>36)$, the tight Gaussian function approximation becomes probably too crude to recover all inner shells and only minima are observed for the K-shell and the most outer shell (see Figure S2).

Some tests for various free atoms and molecules are presented in the next section. Effects of small- and large-core ECPs are also discussed.

Integrated basin populations. When the electron density is a well defined quantity, the basin population is just the integral of the density over the basin volume. In this case, the sum of all basin populations has to recover the total number of electrons. The total basin $\mathrm{N}_{\Omega}$ population can be separated into two parts:

$\mathrm{N}_{\Omega}=\mathrm{N}_{\Omega}^{\mathrm{val}}+\mathrm{N}_{\Omega}^{\mathrm{core}}=\int_{\Omega} \rho(\mathbf{r})^{\mathrm{val}} \mathrm{dr}+\int_{\Omega} \rho(\mathbf{r})^{\text {core }} \mathrm{d} \mathbf{r}$

Where $\mathrm{N}_{\Omega}^{\mathrm{val}}$ and $\mathrm{N}_{\Omega}^{\text {core }}$ are respectively the total valence and core basins populations. $\mathrm{N}_{\Omega}^{\mathrm{val}}$ can be accurately calculated from the sum of elementary overlap integrals over all parallelepipedic cells centered on the grid points belonging to the given basin. ${ }^{[4]}$ As well, for a rectangular cartesian parallelepipedic grid, the elementary core $S^{\text {core }}$ population can be calculated at given grid point $r_{0}$ center of a elementary cell of edges $\Delta r_{1}, \Delta r_{2}$ and $\Delta r_{3}$ :

$\mathrm{S}\left(\mathrm{r}_{\mathrm{o}}\right)^{\text {core }}=\sum_{\mathrm{A}} \frac{Z_{\mathrm{A}}^{\text {core }}}{8} \prod_{\mathrm{i}=1}^{3}\left[\operatorname{erf}\left(\alpha \sqrt{\pi}\left(\frac{\Delta \mathrm{r}_{\mathrm{i}}}{2}+\mathrm{R}_{\mathrm{A}}^{\mathrm{i}}-\mathrm{r}_{\mathrm{o}}^{\mathrm{i}}\right)\right)+\operatorname{erf}\left(\alpha \sqrt{\pi}\left(\frac{\Delta \mathrm{r}_{\mathrm{i}}}{2}-\mathrm{R}_{\mathrm{A}}^{\mathrm{i}}+\mathrm{r}_{\mathrm{o}}^{\mathrm{i}}\right)\right)\right]$

Where $R_{A}^{i}$ are the cartesian coordinates of an atom $A$ and $r^{\text {io }} 0$ the cartesian coordinates of the grid point $r_{0}$. The total core basin population 
$\mathrm{N}_{\Omega}^{\text {core }}$ is calculated as the sum of elementary core integrals $S^{\text {core }}$ over all the cells centered on the grid points belonging to the given basin $\Omega$ :

$$
\mathrm{N}_{\Omega}^{\text {core }}=\int_{\Omega} \rho(\mathbf{r})^{\text {core }} \mathrm{d} \mathbf{r} \approx \sum_{\mathbf{r}_{0} \in \Omega} \mathrm{S}\left(\mathbf{r}_{\mathbf{o}}\right)^{\text {core }}
$$

Within the QTAIM partition, the atomic charge $\mathrm{q}_{\Omega}$ of a topological atom can be then easily computed by:

$$
\mathrm{q}_{\Omega}=\mathrm{Z}_{\Omega}-\mathrm{N}_{\Omega}
$$

IV. Computational details. The B3LYP hybrid functional level with the Gaussian 2009 software $^{[45]}$ was used for all calculations. Extended Hückel calculations ${ }^{[46,47]}$ have been performed with the Gaussian program. The energy-consistent ECPnMDF ECPs were used to replace the $n$ inner-core electrons of the copper $(n=10)$, bromine $(n=10)$, krypton $(n=10)$, iodine $(n=28)$ and gold $(n=60)$ atoms. Their remaining valence electrons were dealt with the triplezeta quality aug-cc-pVTZ-PP basis set. The relativistic effective small-core potential (RECP) of the Stuttgart/Köln group was also tested for the Fe $(n=10)$ and $U(n=60)$ atoms. ${ }^{[48-50]}$ Their remaining valence electrons were dealt with Dunning/Huzinaga full double zeta. The CRENBS and CRENBL basis sets with shape consistent ECP's have been also used. ${ }^{[51,52]}$ In this latter case, large-core RECP were used to mimic the role of the $n$ inner-core electrons of selected atoms: copper $(n=18)$, iron $(n=18)$, iodine $(n=36)$, gold $(n=68)$ and uranium $(n=78)$ atoms. For the lighter atoms, $\mathrm{H}, \mathrm{C}$, or $\mathrm{O}$ the standard all-electron aug-cc-pVTZ or $6-311+G(d, p)$ basis sets were used. The TopChem2 package ${ }^{[53]}$ was used for all QCT analyses presented in the paper. The electron density isosurfaces were displayed by means of the Molekel v4.3 software. ${ }^{[54]}$

V. Results and Discussion. We review here typical examples where the applicability of the methodology is illustrated for a panel of molecular systems selected through the periodic table. The implementation was tested for CuCO $\left(C_{s}\right)^{[55]}$, Ferrocene FeCp $2\left(D_{5 d}\right)^{[56]}, I_{3}$ $\left(\mathrm{C}_{2 v}\right)^{[57]}, \mathrm{AuKrBr}\left(\mathrm{C}_{\infty \mathrm{v}}\right)^{[58]}$ and for the uranyl cation $\mathrm{UO}_{2}{ }^{2+}\left(\mathrm{D}_{\infty h}\right)^{[59]}$ compound. The robustness of the approach for various small-core as well as largecore ECPs was investigated. Table 1 reports the parameters obtained for the core density $(\alpha$, $\mathrm{R}_{\beta}$ ) of core tight functions $\rho(\boldsymbol{r})^{\text {core }}$ as well as the QCT results obtained from the augmented electron density $\rho(\mathbf{r})=\rho(\mathbf{r})^{\text {core }}+\rho(\mathbf{r})^{\text {val }}$ at bond critical points.

\section{Insert Table 1 near here}

The core population of the atom $A \mathrm{~N}^{\beta}{ }_{\mathrm{A}}$ has been computed over the volume of a $\beta$-sphere of radius 1.5 bohr and the total basin $\mathrm{N}_{\mathrm{A}}$ populations are also reported. Numerous different critical points can be identified in the valence or outer-shell regions but only the values of selected topological descriptors located at the nearest BCP of the A atoms are reported, as displayed in Figure 6 . The main goal of this section is to assess whether the topology of core regions is suitably restored by the parametrized tight functions applied to the selected systems. The influence of the augmented electron density on the valence has to be analyzed; this effect should be as small as possible. Using parameters of the core density obtained from small-core Stuttgart/Köln ECP, some tests have also been performed for semiempirical extended Hückel calculations where the core electrons are disregarded. These results are presented in Table 2. A detailed analysis of results presented in Tables 1 and 2 leads to the non-ambiguous following conclusions:

Insert Table 2 near here 
1. Firstly, the results show that core parameters, namely $\mathrm{R}_{\beta}$ and the related exponent $\alpha$, remain almost unchanged between the free atoms and these same atoms involved in related molecular systems. For instance, $\alpha=2.22$ for the iodine free atom I $\left({ }^{2} \mathrm{P}\right)$ when a large-core ECP is used whereas $\alpha=2.21$ for the same atom involved in the $\mathrm{IF}_{3}$ molecule. However, noticeable differences of core parameters are observed when moving from an ECP small-core to a large-core (see Table 1). In any case, the augmented electron density restores the proper features of both the density and the electron localization function in the vicinity of nuclear regions and also at the frontier between the inner and outer atomic shells. The core basin population calculated with the augmented density obtained from a small-core ECP has always been found to be in good agreement with the corresponding density obtained from the all-electron calculation. For instance, a difference of only 0.01 e was found for the core population of $\mathrm{Fe}$ in $\mathrm{FeCp}_{2}$ and the values calculated at the B3LYP/SDD (smallcore) level and the $B 3 L Y P / 6-311+G(d, p)$ level. Using a large-core ECP (CRENBS), this latter difference was found to be $0.19 \mathrm{e}$. This reveals the difference between the wavefunction obtained when a large-core is employed instead of the corresponding all-electron wavefunction.

2. In the regions where the pseudopotential and the all-electron density coincides, one could even likely confirm from the Table 1 that, the core electron densities have a very small effect on the values of topological descriptors evaluated at the location of selected bond critical points (see Figure 6). This statement is true for all typical descriptors computed in this paper, namely, the distance of the BCP $\left(r_{b c p}\right)$ from the nuclear location of the heavy atom, the electron density $\left(\rho_{b}\right)$, the gradient norm of the electron density $\left(\left|\nabla \rho_{b}\right|\right)$, the Laplacian of electron density $\left(\nabla^{2} \rho_{b}\right)$, the ratio of density energies $\left|V_{b}\right| / G_{b}$ and also the value of ELF. Note that the difference between values obtained from the augmented density and the valence density $\rho(\mathbf{r})^{\text {val }}$ are negligible if small-core ECPS are employed and almost unchanged if large-core ECPs are used. For instance, the Laplacian of electron density is only reduced on average by more than 0.001 e.bohr ${ }^{-4}$ for species containing $\mathrm{Cu}$ and $\mathrm{Fe}$ as well as for the heaviest compounds containing the $\mathrm{I}, \mathrm{Au}$ or $\mathrm{U}$ atoms.

3. The robustness of this methodology has also been evaluated in the framework of the extended Hückel semi-empirical calculations where the core density is missing ( $Z_{C}^{\text {core }}=2$ and $Z_{N}^{\text {core }}=2$ and $Z_{O}^{\text {core }}=2$ ). Because pathologies have been identified with the quantum chemical topology analysis a long time ago ${ }^{[7]}$, it is very interesting to explore whether the tight Gaussian functions are also able to restore the proper features. All results for $\mathrm{C}_{2} \mathrm{H}_{4}$, the adenine, $\mathrm{NH}_{2} \mathrm{OH}$ and the furan molecules are gathered in Table 2. The plots of electron densities of $\mathrm{C}_{2} \mathrm{H}_{4}$ and adenine are displayed in Figures 7 and 8. As observed in Figure 7 (left), pathologies appear in the gradient vectors field of the electron density of $\mathrm{C}_{2} \mathrm{H}_{4}$, especially located in the regions near the carbon atoms. Indeed, gradient vectors seem to be wrongly oriented from the nuclear carbon centers to valence regions and no bond critical point $(3,-1)$ has been found between the two carbon atoms. In contrast (Figure 7, right), using the core parameters obtained from a Hartree- 
Fock/SDD calculation ( $Z_{C}^{\text {core }}=2$ and $Z_{N}^{\text {core }}$ $=2$ ), the topology seems to be restored for augmented densities. Indeed, atomic basins have been now clearly identified for carbon atoms and a typical saddle point $(3,-1)$ has been found at the middle of the $\mathrm{C}-\mathrm{C}$ bond. In a similar manner, the plot augmented density and the location of the bcp obtained at the Hückel level of theory along a selected $\mathrm{N}-\mathrm{C}$ bond of the adenine molecule is depicted in Figure 8. Interestingly, the latter (Figure 8 right, red line) appears close to those obtained at the B3LYP/6-311G(2d) level of theory which is taken as reference (Figure 8 right, blue line). Without curing, many spurious critical points are found in the inner regions and also in the border regions with the valence. The augmented density analysis shows maxima on the nuclear locations of $C$ and $\mathrm{N}$ atoms which allow us to soundly restore the topological properties throughout the whole molecular space. Bond critical points $(3,-1)$ have been indeed found in valence regions close to the middle of $\mathrm{N}-\mathrm{C}$ and $\mathrm{C}-\mathrm{C}$ bonds. But more important are the trends drawn from the numbers. As shown in Table 2, previously outlined trends regarding the properties calculated at the BCP in Table 1 can be extended to the Hückel level of theory. Indeed, the features of descriptors at $\mathrm{BCP}$ remain in reasonable agreement with the Hartree-Fock/SDD level of theory. For example, for the $\mathrm{N}-\mathrm{C}$ bonds as well as for the $\mathrm{N}-\mathrm{O}$ bond, the electron density computed at the BCP $\left(\rho_{b}\right)$ is notably strong, $(0.25-0.30$ e bohr 3 on average), the Laplacian of the density $\left(\nabla^{2} \rho_{\mathrm{b}}\right)$ exhibits a (small) negative value, while the ratio $\mid \mathrm{V}(\mathbf{r})_{\mathrm{b}} \mathrm{l} / \mathrm{G}(\mathbf{r})_{\mathrm{b}}$ is higher than 2.0 and ELF goes to 1 . These features are commonly associated with a typical shared-shell (covalent) interaction.

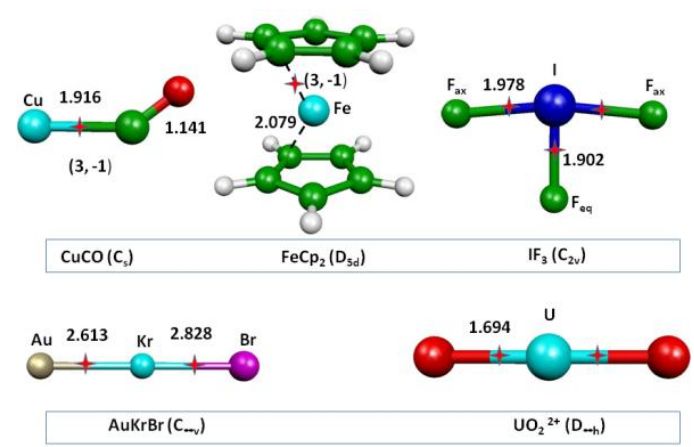

Figure 6. Optimized structural parameters ( $\AA$ ) of compounds given in Table 1 obtained at the B3LYP level of theory. The location of the bond critical points given in Table 1 and discussed in the paper, are displayed with a red dagger.
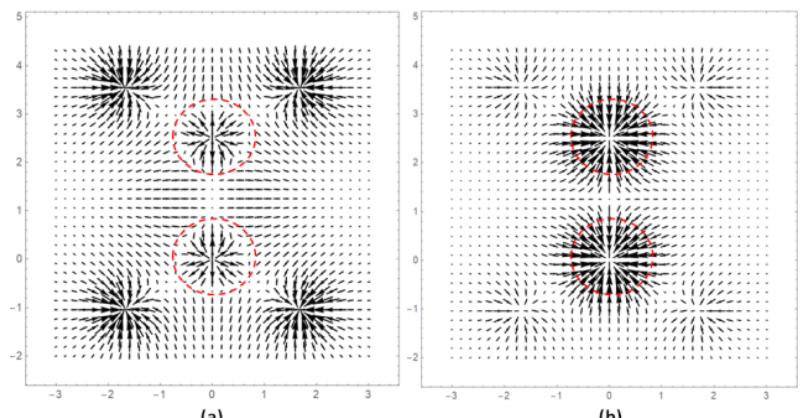

(a)

(b)

Figure 7. The Gradient vector field of the electron density displayed in the molecular plane of the $\mathrm{C}_{2} \mathrm{H}_{4}$ molecule optimized at the extended Hückel level of theory.

(a) Valence electron density $\rho(\mathbf{r})^{\mathrm{val}}$.

(b) Augmented electron density $\rho(\mathbf{r})=\rho(\mathbf{r})^{\text {core }}+\rho(\mathbf{r})^{\text {val }}$.

Core parameters: $Z_{C}^{\text {core }}=2$ electrons, $R_{\beta}=0.797$ bohr and $\alpha=0.866$ bohr $^{-1}$. The red circles stake out the carbon topological atoms.

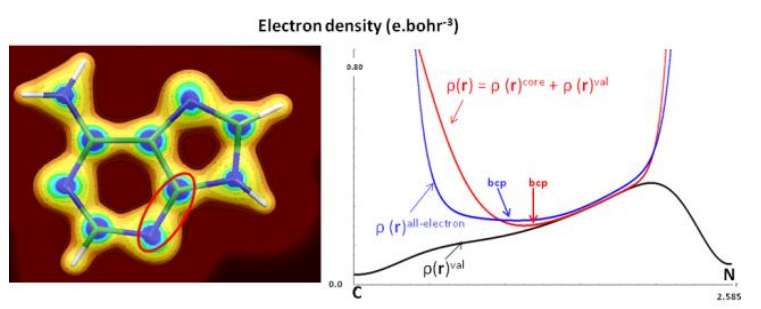

Figure 8. Left: Augmented Electron density $\rho(\mathbf{r})=\rho(\mathbf{r})^{\text {core }}+$ $\rho(r)^{\text {val }}$ plot $\left(\right.$ e.bohr $\left.{ }^{-3}\right)$ for the $\mathrm{C}_{5} \mathrm{H}_{5} \mathrm{~N}_{5}$ molecule optimized at the extended Hückel level of theory. Blue regions are high values of the density (left).

Right: Electron density plot displayed along the selected N$\mathrm{C}$ bond surrounded by a circle. Color code: black: valence Hückel electron density $\rho(\mathbf{r})^{\mathrm{val}}$. Blue: all-electron density calculated at the B3LYP/6-311G(2d) level of theory. The bcp is located at 1.117 bohr from the carbon atom. Red: augmented electron density $\rho(\mathbf{r})$. The bcp is located at 1.243 bohr from the carbon atom. 
Core parameters: C: $Z_{C}^{\text {core }}=2$ electrons, $R_{\beta}=0.856$ bohr, $\alpha=$ 0.807 bohr $^{-1} . \mathrm{N}: \mathrm{Z}_{\mathrm{N}}^{\text {core }}=2$ electrons, $\mathrm{R}_{\beta}=0.482 \mathrm{bohr}, \alpha=$ 2.179 bohr $^{-1}$.

\section{Concluding remarks}

Beyond considerations regarding the analysis and the understanding of bonding schemes when pseudopotentials are used, this study has demonstrated the puzzling robustness and the fluency of parametrized tight Gaussian functions to restore the core density and the proper profiles of the electron localization function in the inner atomic regions. Only small modifications were found on the values of investigated topological descriptors when changing from small-core to large-core ones, or when moving from small basis set to extended ones. Also, it has been confirmed that the QCT analysis is quite suitable for systems containing heavy elements in the context of semi-empirical calculations when core densities have been restored in this way. Without this healing, the QCT analysis remains strongly hampered by the spurious topology of the gradient vectors field. Finally, the work shows how this methodology can be easily implemented at different nonrelativistic or relativistic levels of theory (HF, semi-empirical or DFT) or whatever the quantum program used. An extension of this work to any correlated wavefunction can also be planned using approximated electron densities and KED defined in terms of natural orbitals. ${ }^{[60,61]}$ To conclude, their indisputable utility opens up opportunities for helping chemists to apply the QCT tools when systems containing heavy atoms are used. 


\section{References}

1. S. Huzinaga, A. A. Cantu, J. Chem. Phys 1971, 55, 5543.

2. J. C. Phillips, L. Kleinman, Phys. Rev. 1959, 116, 287. PR

3. M. Dolg, X. Cao, Chem. Rev. 2012, 112, 403.

4. X. Cao, M. Dolg, WIREs Computational Molecular Science 2011, 1, 200.

5. S. F. Vyboishchikov, A. Sierraalta, G. Frenking, J. Comput. Chem. 1997, 18, 416.

6. A. Sierraalta, F. Ruette, Int. J. Quantum Chem 1996, 60, 1015.

7. M. HÔ, H. Schmider, K. E. Edgecombe, V. H. Smith Jr, Int. J. Quantum Chem 1994, 52, 215.

8. A. D. Becke, K. E. Edgecombe, J. Chem. Phys 1990, 92, 5397.

9. R. F. W. Bader, Chem. Rev. 1991, 91, 893.

10. R. F. W. Bader. Atoms in Molecules: A Quantum Theory; Oxford University Press: New York, USA, 1994.

11. D. Tiana, E. Francisco, M. A. Blanco, A. M. Pendás, J. Phys. Chem. A 2009, 113, 7963.

12. P. Maxwell, Á. M. Pendás, P. L. A. Popelier, PCCP 2016, 18, 20986.

13. N. Saeed Saadi, Advances in Physics Theories and Applications 2013, 25, 92.

14. J. Cioslowski, P. Piskorz, P. Rez, J. Chem. Phys 1997, 106, 3607.

15. J. Cioslowski, P. Piskorz, Chem. Phys. Lett. 1996, 255, 315.

16. T. A. Keith, M. J. Frisch, J. Phys. Chem. A 2011, 115, 12879.

17. W. Zou, Z. Cai, J. Wang, K. Xin, J. Comput. Chem. 2018, 39, 1697.

18. M. Amaouch, E. Renault, G. Montavon, N. Galland, J. Pilmé. In Applications of Topological Methods in Molecular Chemistry; Chauvin, R.; Lepetit, C.; Silvi, B.; Alikhani, E., Eds.; Springer International Publishing: Cham, 2016, p 553.

19. A. M. Pendás, E. Francisco, J. Chem. Theory Comput. 2019, 15, 1079.

20. P. L. A. Popelier. In Intermolecular Forces and Clusters I; Wales, D. J., Ed.; Springer Berlin Heidelberg: Berlin, Heidelberg, 2005, p 1.

21. P. Fuentealba, E. Chamorro, J. C. Santos. In Theoretical and Computational Chemistry; Toro-Labbé, A., Ed.; Elsevier, 2007, p 57.

22. J. Pilmé, E. Renault, F. Bassal, M. Amaouch, G. Montavon, N. Galland, J. Chem. Theory Comput. 2014, 10, 4830.

23. J. S. M. Anderson, J. I. Rodríguez, P. W. Ayers, D. E. Trujillo-González, A. W. Götz, J. Autschbach, F. L. Castillo-Alvarado, K. Yamashita, Chem. Eur. J. 2019, 25, 2538.

24. J. Pilmé, E. Renault, T. Ayed, G. Montavon, N. Galland, J. Chem. Theory Comput. 2012, 8, 2985.

25. A. Savin, O. Jepsen, J. Flad, O. K. Andersen, H. Preuss, H. G. von Schnering, Angew. Chem. Int. Ed. Engl. 1992, 31, 187.

26. B. Silvi, A. Savin, Nature 1994, 371, 683.

27. T. Saue, K. Faegri, O. Gropen, Chem. Phys. Lett. 1996, 263, 360.

28. J. Graton, S. Rahali, J.-Y. Le Questel, G. Montavon, J. Pilmé, N. Galland, PCCP 2018, 20, 29616.

29. F. W. Biegler-Konig, T. T. Nguyen-Dang, Y. Tal, R. F. W. Bader, A. J. Duke, J. Phys. B: At., Mol. Opt. Phys. 1981, 14, 2739.

30. P. L. A. Popelier, J. Comput. Chem. 2018, 39, 604.

31. P. L. A. Popelier, J. Phys. Chem. A 2011, 115, 13169.

32. R. M. Corless, G. H. Gonnet, D. E. G. Hare, D. J. Jeffrey, D. E. Knuth, Advances in Computational Mathematics 1996, 5, 329.

33. R. F. W. Bader, P. M. Beddall, P. E. Cade, J. Am. Chem. Soc. 1971, 93, 3095.

34. R. F. W. Bader, H. Essén, J. Chem. Phys 1984, 80, 1943.

35. D. Cremer, E. Kraka, Angew. Chem. Int. Ed. Engl. 1984, 23, 627.

36. C. F. v. Weizsäcker, Z. Phys. 1935, 96, 431.

37. D. A. Kirzhnits, JETP 1957, 5, 64.

38. V. Tsirelson, A. Stash, Chem. Phys. Lett. 2002, 351, 142.

39. P. W. Ayers, J. Chem. Sci. 2005, 117, 441.

40. B. Silvi, R. J. Gillespie, C. Gatti. In Comprehensive Inorganic Chemistry II (Second Edition); Reedijk, J.; Poeppelmeier, K., Eds.; Elsevier: Amsterdam, 2013, p 187.

41. M. Kohout, A. Savin, Int. J. Quantum Chem 1996, 60, 875. 
42. J. Pilmé, J. Comput. Chem. 2017, 38, 204.

43. J. C. Santos, W. Tiznado, R. Contreras, P. Fuentealba, J. Chem. Phys 2004, 120, 1670.

44. S. Noury, X. Krokidis, F. Fuster, B. Silvi, Comput. Chem. 1999, 23, 597.

45. M. J. Frisch, G. W. Trucks, H. B. Schlegel, G. E. Scuseria, M. A. Robb, J. R. Cheeseman, G. Scalmani, V. Barone, B. Mennucci, G. A. Petersson, H. Nakatsuji, M. Caricato, X. Li, H. P. Hratchian, A. F. Izmaylov, J. Bloino, G. Zheng, J. L. Sonnenberg, M. Hada, M. Ehara, K. Toyota, R. Fukuda, J. Hasegawa, M. Ishida, T. Nakajima, Y. Honda, O. Kitao, H. Nakai, T. Vreven, J. A. Montgomery, J. E. P. Jr., F. Ogliaro, M. Bearpark, J. J. Heyd, E. Brothers, K. N. Kudin, V. N. Staroverov, R. Kobayashi, J. Normand, K. Raghavachari, A. Rendell, J. C. Burant, S. S. Iyengar, J. Tomasi, M. Cossi, N. Rega, J. M. Millam, M. Klene, J. E. Knox, J. B. Cross, V. Bakken, C. Adamo, J. Jaramillo, R. Gomperts, R. E. Stratmann, $\mathrm{O}$. Yazyev, A. J. Austin, R. Cammi, C. Pomelli, J. W. Ochterski, R. L. Martin, K. Morokuma, V. G. Zakrzewski, G. A. Voth, P. Salvador, J. J. Dannenberg, S. Dapprich, A. D. Daniels, O. Farkas, J. B. Foresman, J. V. Ortiz, J. Cioslowski, D. J. Fox, Gaussian, Inc., Wallingford CT, 2009.

46. E. Hückel, Z. Phys. 1930, 60, 423.

47. E. Hückel, Z. Phys. 1931, 70, 204.

48. M. Dolg, U. Wedig, H. Stoll, H. Preuss, J. Chem. Phys 1987, 86, 866.

49. X. Cao, M. Dolg, J. Mol. Struct. : THEOCHEM 2002, 581, 139.

50. X. Cao, M. Dolg, H. Stoll, J. Chem. Phys 2003, 118, 487.

51. M. M. Hurley, L. F. Pacios, P. A. Christiansen, R. B. Ross, W. C. Ermler, J. Chem. Phys 1986, 84, 6840.

52. L. Fernandez Pacios, P. A. Christiansen, J. Chem. Phys 1985, 82, 2664.

53. D. Kozlowski, J. Pilmé, J. Comput. Chem. 2011, 32, 3207. Available at: http://www.lct.jussieu.fr/pagesperso/pilme/topchempage.html (accessed on November 25, 2019).

54. P. Flukiger, H. P. Luthi, S. Portmann, J. Weber, Manno, Switzerland, 2002.

55. J. Pilme, B. Silvi, M. E. Alikhani, J. Phys. Chem. A 2003, 107, 4506.

56. L. J. Farrugia, C. Evans, D. Lentz, M. Roemer, J. Am. Chem. Soc. 2009, 131, 1251.

57. M. Amaouch, D.-C. Sergentu, D. Steinmetz, R. Maurice, N. Galland, J. Pilmé, J. Comput. Chem. 2017, 38, 2753.

58. E. Makarewicz, A. J. Gordon, S. Berski, J. Phys. Chem. A 2015, 119, 2401.

59. V. Vallet, U. Wahlgren, I. Grenthe, J. Phys. Chem. A 2012, 116, 12373.

60. A. S. Szabo, N. S. Ostlund. Modern Quantum Chemistry - Introduction to advanced electronic structure theory: New York, 1987.

61. F. Feixas, E. Matito, M. Duran, M. Solà, B. Silvi, J. Chem. Theory Comput. 2010, 6, 2736.

\footnotetext{
${ }^{1}$ Sorbonne Université, CNRS, Laboratoire de Chimie Théorique CC 137 - 4, place Jussieu F. 75252 PARIS CEDEX 05 - France
} 